\title{
Are Cholinesterase Inhibitors Effective in Improving Balance in Parkin- son's Disease?
}

\author{
Hiller ALP ${ }^{1,2}$, Nutt JG', Mancini $\mathbf{M}^{1 *}$, Horak FB ${ }^{1,2}$, Kareus $\mathbf{S}^{1-3}$, Schoneburg $\mathrm{BM}^{1,2,4}$ and Chung KA $\mathrm{A}^{1,2}$ \\ ${ }^{1}$ Department of Neurology, Oregon Health and Science University, Portland, Oregon, USA \\ ${ }^{2}$ Department of Neurology, VA Portland Health Care System, Portland, Oregon, USA \\ ${ }^{3}$ Department of Neurology, Grand Junction, Colorado, USA \\ ${ }^{4}$ Department of Neurology, Glenview, Illinois, USA
}

\begin{abstract}
Background: Cholinesterase inhibitors have been reported to reduce falls in a double blind pilot study. The mechanism by which cholinesterase inhibitors reduce falls is unknown.

Methods: A pilot, double-blind, placebo-controlled, crossover study examined the effects of donepezil on posturography and frontal executive function. Participants received 6 weeks of treatment with placebo and donepezil in random order, separated by a month washout. Inclusion criteria were an MMSE $\geq 27$ and balance impairment on clinical and sensory orientation posturography (SOT) assessments.

Results: Ten participants completed the study. Donepezil improved postural sway in SOT condition 4 (C4, eyes open, sway referenced surface) $(p<0.03)$. The change seen in executive performance (measured by the Trail B-A time) when on donepezil correlated with improvement in SOT C4, $r=0.80, p=0.001$.

Conclusion: Cholinesterase inhibitors improved two functions related to fall risk, standing balance on an unstable surface and executive set-switching in subjects with PD. We hypothesized that the cholinesterase inhibitor, donepezil, affects fall risk by improving sensory orientation set-switching for balance related to improvements in executive setswitching.
\end{abstract}

\section{Background}

Most falls in Parkinson's disease (PD) appear to be due to a primary balance dysfunction rather than an environmental factor [1]. Accumulating evidence suggests that balance dysfunction cannot only be attributed to changes in the dopaminergic system that seems to be responsible for most of the other motor symptoms in PD [1]. The interventions often helpful in improving other PD symptoms, dopaminergic medications and deep brain stimulation, do not consistently improve balance and falls [2,3]. Degeneration in other brain areas, such as cholinergic cortex, the adrenergic locus coeruleus or the cholinergic/glutaminergic pedunculopontine nucleus may be the origin of the balance problems associated with PD [4].

Cholinesterase inhibitors have a positive impact on global assessments, cognitive function, behavioral disturbances, and activities of daily living in persons with PD with Dementia, Lewy Body Dementia, and PD with Cognitive impairment but without dementia (CIND) [5]. A pooled estimate of therapeutic benefit of cholinesterase inhibitors on cognitive function was found with a standard mean difference (SMD) of -0.34 ( $95 \%$ CI -0.46 to $-0.23, p<0.00001$ ) versus placebo. A study enrolling 23 subjects in a cross-over design, showed a reduction in falls in persons with PD without dementia when taking donepezil for six weeks. Fall frequency per day decreased from $0.25 \pm 0.08$ on placebo to $0.13 \pm 0.03$ on donepezil $(p<0.05)[6]$.

It is not clear the exact role cholinergic function plays in gait, balance, and falls. It is clear that functional walking without falls requires executive function, such as the ability to switch attention quickly [7]. The relationship between cholinergic function and falls has been demonstrated with PET imaging [8]. The cholinergic system, specifically the pendunculopontine nucleus (PPN), may have direct effects on gait and balance. Loss of PPN cells is correlated with balance performance and may affect attention [9-11]. There are also extensive cholinergic projections to the cerebral cortex with the nucleus basalis of Meyert that suplies the marjority of input to the frontoparietal attention network [12]. The frontoparietal attention network has been implicated as a mediator of space-based attention that may be critical for switching attention among sensory systems for spatial orientation for postural control [13]. The cholinergic system, in this case, may play an indirect role on balance via executive function. In fact, patients with executive function deficits show more balance and gait deficits and falls [14]. It is possible that cholinesterase inhibitors could help reduce falls through direct effects on balance and/or via indirect effects on cognition.

The current work is a pilot study with a primary aim to determine the effects of donepezil (a centrally acting cholinesterase inhibitor) on cognition and balance in non-demented persons with PD compared to a placebo.

\section{Design/Methods}

\section{Participants}

Participants were recruited though Oregon Health and Sciences University (OHSU) movement disorders clinic. The Human Subjects Institutional Boards of OHSU approved the study. All individuals provided informed consent. The study was a randomized, doubleblind, placebo controlled, crossover design. Participants received 6

*Corresponding author: Martina Mancini, Department of Neurology, OHSU, Mail Code OP32, 3181 SW Sam Jackson Park Road, P.O. Box: 97239, Portland, USA, Tel: 5033460842; Fax: 5034949059; E-mail: mancinim@ohsu.edu

Received September 08, 2015; Accepted September 08, 2015; Published September 12,2015

Citation: Hiller ALP, Nutt JG, Mancini M, Horak FB, Kareus S, et al. (2015) Are Cholinesterase Inhibitors Effective in Improving Balance in Parkinson's Disease? J Neurol Disord S2: 002. doi:10.4172/2329-6895.S2-002

Copyright: ( 2015 Hiller ALP, et al. This is an open-access article distributed under the terms of the Creative Commons Attribution License, which permits unrestricted use, distribution, and reproduction in any medium, provided the original author and source are credited. 
weeks of treatment with either placebo or donepezil with assessments at the beginning and end of the 6-week period, followed by a month washout, then 6 weeks of the other treatment again with assessments at the beginning and end.

Inclusion criteria were 1) idiopathic PD (as determined by UK Brain Bank Criteria) with a Hoehn and Yahr score of 2 to 4,2) treated with levodopa for at least a year and on a stable antiparkinsonian regiment for at least one month, 3) abnormal dynamic posturography on screening with a composite score of 70 or less. Exclusion criteria were 1) dementia, defined as an MMSE<27, 2) other medical conditions affecting gait 3) inability to stand unassisted, 4) already on cholinesterase inhibitors or anticholinergic medications, or 5) other medical or psychiatric comorbidities that could interfere with compliance or safety.

\section{Data collection}

Balance was measured using the sensory organization test (SOT) on the NeuroCom Balance Master Clinical Research System platform (Neurocom International, Inc), which tests sway in 6 sensory conditions: 1) eyes open, 2) eyes closed, 3) sway referenced visual surround with a stable platform, 4) sway referenced platform with eyes open and 5) sway referenced visual surround and platform. Center of Pressure $(\mathrm{CoP})$ was calculated from the force plate recordings. Forces and moments were recorded at $100 \mathrm{~Hz}$ sampling frequency, and data were exported and analyzed with a custom algorithm in Matlab (MathWorks, Inc). Unlike commercial posturography, we measured medio-lateral postural sway dispersion because it is most highly related to falls $[15,16]$.

In addition, Parkinson's severity scales included United Parkinson's Disease Rating Scale Motor Section (UPDRS-III) and cognitive testing included the MMSE at baseline, Stroop, and Trail Making Tests. A general physical and neurological exam, heart rate, and blood pressure measures where completed at each visit. During the active treatment phase subjects were given donepezil $5 \mathrm{mg}$ for three weeks and if tolerated, increased to $10 \mathrm{mg}$ for weeks four through six.

A total of twenty one subjects were enrolled in the study; ten withdrew because of adverse events and one subject did not meet enrollment criteria (MMSE was too low). Five withdrawals were related to the study drug with 4 due to nausea, vomiting, or gastrointestinal discomfort and one who felt freezing and balance was worse after starting the study drug. One dropped out after a possible transient ischemic attack, one after a serious fall, two due to scheduling concerns, and one due to worsened radicular pain.

Ten subjects ( 5 male, 5 female, mean age: $70 \pm 6$ years, mean UPDRS motor score: $24 \pm 7$, mean PD disease duration: $10.5 \pm 8$ years, mean MMSE at baseline: $29.2 \pm 0.4$, Stroop conflict time: $105 \pm 48 \mathrm{~s}$, and mean Trails B-A difference: $59 \pm 32 \mathrm{~s}$ ) completed both phases of the study and their data were used in the analysis.

\section{Data analysis}

The Root Mean Square distance (RMS) was calculated for condition 1-6 of the SOT, in both the antero-posterior (AP) and medio-lateral (ML) direction from the COP measurements, as measure of sway dispersion. Due to the small sample size, the difference in RMS, UPDRS motor, and cognitive function between the end and the beginning of each treatment phase (placebo or active drug) were calculated and we used a Student's t-test to assess if the difference significantly differed from 0 (where 0 represents no change). A Pearson product-moment correlation was used to assess the relationship between balance and cognitive performances. Data analysis and statistics were performed by a blinded researcher $(\mathrm{MM})$.

\section{Results}

Balance performance improved with donepezil. Specifically, the change in ML RMS in condition 4 of the SOT, during the donepezil phase (beginning-end), was significantly different from zero $(p=0.03)$, reflecting a significant decrease in the ML RMS. In contrast ML RMS did not change during the placebo phase (beginning-end), as depicted in Figure 1, left panel. Individual subject results are showed in Figure 1, right panel. Specifically, out of 10 participants, 4 improved more than $40 \%, 4$ between $15 \%$ and $30 \%$, and 2 didn't show changes in postural sway during the active phase of the study. There were no significant differences seen in the AP RMS, nor in conditions $1-3,5$ or 6 of the SOT, in either the donepezil or placebo phase.

A significant correlation between improvement in the Trail B-A time and improvement in the ML RMS was found in the active phase, $\mathrm{r}=0.8, p=0.001$ (Figure 2). In addition, the change in the Stroop conflict time and trails $\mathrm{B}-\mathrm{A}$ were $-13.3 \pm 24 \mathrm{~s}$ and $5.4 \pm 44 \mathrm{~s}$ after the placebo phase versus $-14 \pm 23 \mathrm{~s}$ and $-13.8 \pm 20$ s after the donepezil phase. None of those changes reached statistical significance.

\section{Conclusion}

In this small, pilot study, we did find that the cholinesterase inhibitor, donepezil, improved a measure of balance in persons with PD without dementia, and that there was a relationship between changes in executive function and changes in balance. These findings are in keeping with previous intervention studies that have showed cholinesterase inhibitors improve cognitive function in persons with PD [17] and Alzheimer's disease [18,19]. The improvement in the Trailmaking executive function might suggest that donepezil improved the ability to rapidly switch attention between letters and numbers.

The relationship between balance improvement and improvement in executive function may be causal or parallel effects. It is possible that attentional set-switching cognitive mechanisms are directly involved in switching attention among sensory systems for postural control, such as between somatosensory and vision in Condition 4 of the SOT. It is also possible that cholinergic innervation affects both executive function and balance in parallel and both factors influence fall risk. Perhaps the fall reduction seen in the study of Chung et al. could be attributable to improvements in both balance and executive function since an increase in mediolateral postural sway has been associated with falls $[20,21]$ and cognitive impairments have been associated with falls [14].
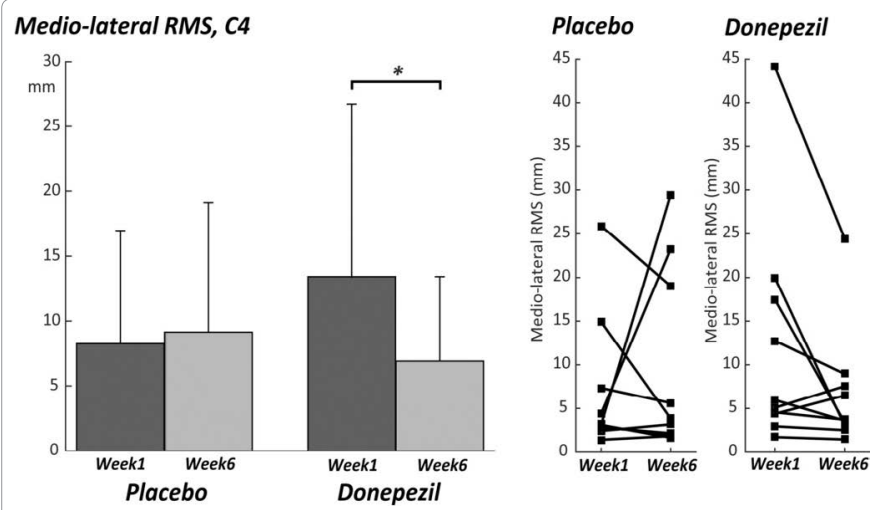

Figure 1: Left panel: Mean \pm STD of the medio-lateral RMS during condition 4 of the Sensory Organization Test at the beginning and after 6-weeks of treatment with a placebo and Donepezil. Right panel: Medio-lateral RMS in single subjects at the beginning and after 6-weeks of treatment with a placebo and Donepezil. 


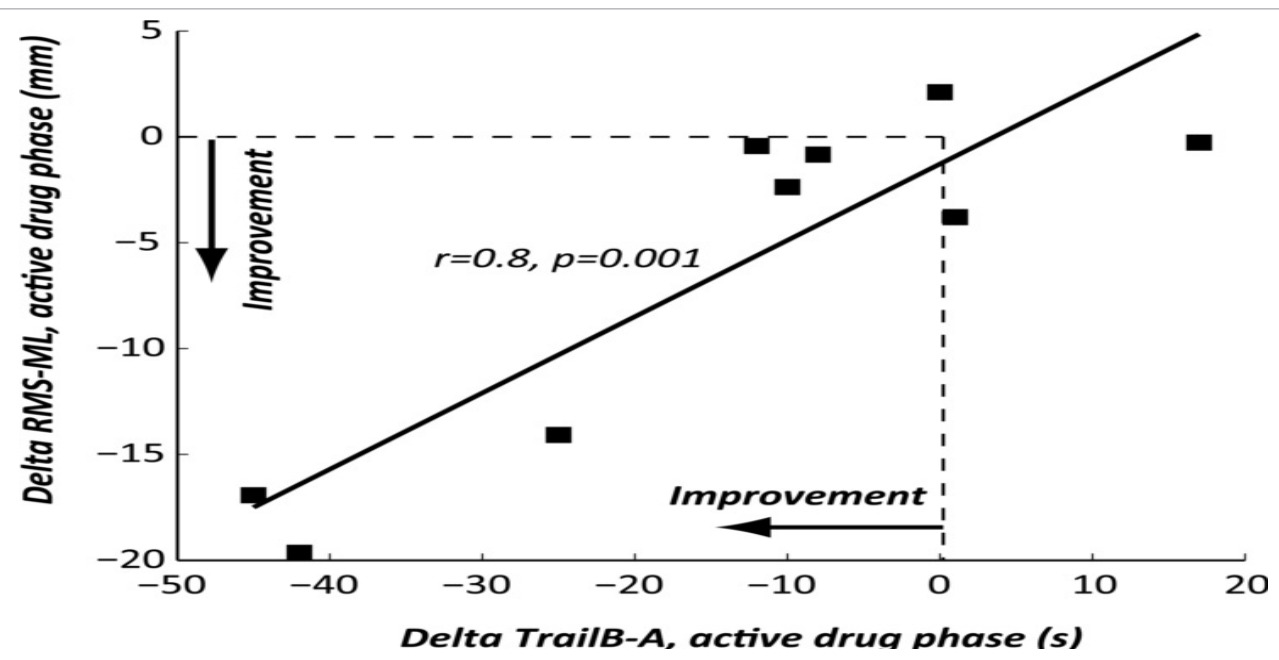

Figure 2: Association between the change in executive function (measured by the TrialB-A time) and the change in medio-lateral sway (measured by the ML-RMS in condition 4 of the SOT) during the 6-weeks of active drug treatment.

Increased postural sway has been associated with thalamic cholinergic denervation in subjects with PD [22]. The effects specifically on SOT condition 4 support a role for the PPN-thalamic cholinergic projections in sensory integration [22]. The decreased ML RMS in the active phase of the study during one of the most problematic balance conditions for PD is when the sensory integration is between somatosensory altered information and visual-vestibular information. Control of standing balance depends on the ability to switch attentional focus among visual, proprioceptive, and vestibular systems, as needed when the environmental conditions change [23]. Previous studies indicated that integration of somatosensory with visual information is affected in PD [24], and that patients with PD show excessive postural sway in conditions with limited or inappropriate sensory feedback $[25,26]$. Therefore, we hypothesized that cholinergic treatment in PD may ameliorate postural instability, particularly in condition 4 , in which somatosensory information is altered. In addition, the conditions including standing on a moving support surface have been associated with increased falls [20]. This is promising for future studies and suggests that cholinergic treatment in PD subjects may ameliorate postural instability and reduce falls through improved postural sensory integration function.

There are multiple limitations of this study. Our dropout rate was very high, which is concerning but seems atypical relative to other studies with donepezil in PD. Dubois completed a large study with 195 randomized to donepezil $5 \mathrm{mg}$ and another 182 to donepezil $10 \mathrm{mg}$. Dropout rates in the active arms were $24.1 \%$ and $23.6 \%$ versus $17.9 \%$ in the placebo group with adverse events reported in $13.8 \%, 17 \%$, and $11 \%$ respectively [17].

Also we only saw changes in one of the SOT conditions. This may represent a bias due to our small population or it could support previous results finding that poorer postural control has been reported in PD in situations where somatosensory information was impaired, perhaps reflecting difficulties to use or integrate visual or vestibular feedback, as reflected in condition 4 of the SOT [24,27]. This small pilot study is meant to inform future study direction and design. The improvements seen in certain measures of balance are promising and suggest further investigation is certainly warranted and should include in-depth measures of balance, specifically SOT4, and cognitive function as well as perhaps some dual task testing. With a lack of pharmacological or surgical treatments to improve balance and falls in PD these results are certainly exciting and deserve further investigation.

\section{Acknowledgments}

This study had support from VA Career Development Award, PADRECC fellowships, and the National Institute of Aging. We thank Michael Fleming for data collection.

\section{References}

1. Bloem BR, van Vugt JP, Beckley DJ (2001) Postural instability and falls in Parkinson's disease. Adv Neurol 87: 209-223.

2. Bloem BR, Beckley DJ, van Dijk JG, Zwinderman AH, Remler MP et al. (1996) Influence of dopaminergic medication on automatic postural responses and balance impairment in Parkinson's disease. Mov Disord 11: 509-521.

3. Weaver FM, Follett K, Stern M, Hur K, Harris C, et al. (2009) Bilateral deep brain stimulation vs best medical therapy for patients with advanced Parkinson disease: a randomized controlled trial. JAMA 301: 63-73.

4. Bloem BR, Hausdorff JM, Visser JE, Giladi N (2004) Falls and freezing of gait in Parkinson's disease: a review of two interconnected, episodic phenomena. Mov Disord 19: 871-884.

5. Rolinski M, Fox C, Maidment I, McShane R (2012) Cholinesterase inhibitors for dementia with Lewy bodies, Parkinson's disease dementia and cognitive impairment in Parkinson's disease. Cochrane Database Syst Rev 3: CD006504.

6. Chung KA, Lobb BM, Nutt JG, Horak FB (2010) Effects of a central cholinesterase inhibitor on reducing falls in Parkinson disease. Neurology 75 1263-1269.

7. Amboni M, Barone P, Hausdorff JM (2013) Cognitive contributions to gait and falls: evidence and implications. Mov Disord 28: 1520-1533.

8. Bohnen NI, Müller ML, Koeppe RA, Studenski SA, Kilbourn MA, et al. (2009) History of falls in Parkinson disease is associated with reduced cholinergic activity. Neurology 73: 1670-1676.

9. Mena-Segovia J, Bolam JP, Magill PJ (2004) Pedunculopontine nucleus and basal ganglia: distant relatives or part of the same family? Trends Neurosc 27: 585-588.

10. Pahapill PA, Lozano AM (2000) The pedunculopontine nucleus and Parkinson's disease. Brain 123: 1767-1783.

11. Rinne JO, Ma SY, Lee MS, Collan Y, Röyttä M (2008) Loss of cholinergic neurons in the pedunculopontine nucleus in Parkinson's disease is related to disability of the patients. Parkinsonism Relat Disord 14: 553-557.

12. Coyle JT, Price DL, DeLong MR (1983) Alzheimer's disease: a disorder of cortical cholinergic innervation. Science 219: 1184-1190.

13. Scolari M, Seidl-Rathkopf KN, Kastner S (2015) Functions of the human frontoparietal attention network: Evidence from neuroimaging. Current Opinion in Behavioral Sciences 1: 32-39.

14. Smulders K, Esselink RA, Cools R, Bloem BR (2014) Trait impulsivity is associated with the risk of falls in Parkinson's disease. PLoS One 9: e91190. 
Citation: Hiller ALP, Nutt JG, Mancini M, Horak FB, Kareus S, et al. (2015) Are Cholinesterase Inhibitors Effective in Improving Balance in Parkinson's Disease? J Neurol Disord S2: 002. doi:10.4172/2329-6895.S2-002

Page 4 of 4

15. Bauer CM, Gröger I, Rupprecht R, Marcar VL, Gaßmann KG (2015) Prediction of future falls in a community dwelling older adult population using instrumented balance and gait analysis. Z Gerontol Geriatr.

16. Piirtola M, Era P (2006) Force platform measurements as predictors of falls among older people - a review. Gerontology 52: 1-16

17. Dubois B, Tolosa E, Katzenschlager R, Emre M, Lees AJ, et al. (2012) Donepezil in Parkinson's disease dementia: a randomized, double-blind efficacy and safety study. Mov Disord 27: 1230-1238.

18. Montero-Odasso M, Muir-Hunter SW, Oteng-Amoako A, Gopaul K, Islam A, et al. (2015) Donepezil improves gait performance in older adults with mild Alzheimer's disease: a phase II clinical trial. J Alzheimers Dis 43: 193-199.

19. Seltzer B, Zolnouni P, Nunez M, Goldman R, Kumar D, et al. (2004) Efficacy of donepezil in early-stage Alzheimer disease: a randomized placebo-controlled trial. Arch Neurol 61: 1852-1856.

20. Frenklach A, Louie S, Koop MM, Bronte-Stewart H (2009) Excessive postural sway and the risk of falls at different stages of Parkinson's disease. Mov Disord 24: 377-385.

21. Nantel J, McDonald JC, Bronte-Stewart H (2012) Effect of medication and STNDBS on postural control in subjects with Parkinson's disease. Parkinsonism
Relat Disord 18: 285-289.

22. Muller ML, Albin RL, Kotagal V, Koeppe RA, Scott PJ, et al. (2013) Thalamic cholinergic innervation and postural sensory integration function in Parkinson's disease. Brain 136: 3282-3289.

23. Peterka RJ (2003) Simplifying the complexities of maintaining balance. IEEE Eng Med Biol Mag 22: 63-68.

24. Colnat-Coulbois S, Gauchard GC, Maillard L, Barroche G, Vespignani $H$, et al (2011) Management of postural sensory conflict and dynamic balance control in late-stage Parkinson's disease. Neuroscience 193: 363-369.

25. Horak FB, Nutt JG, Nashner LM (1992) Postural inflexibility in parkinsonian subjects. J Neurol Sci 111: 46-58.

26. Bronte-Stewart HM, Minn AY, Rodrigues K, Buckley EL, Nashner LM (2002) Postural instability in idiopathic Parkinson's disease: the role of medication and unilateral pallidotomy. Brain 125: 2100-2114.

27. Shivitz N, Koop MM, Fahimi J, Heit G, Bronte-Stewart HM (2006) Bilateral subthalamic nucleus deep brain stimulation improves certain aspects of postural control in Parkinson's disease, whereas medication does not. Mov Disord 21: 1088-97.
This article was originally published in a special issue, Microglia and Synaptic Reorganization handled by Editor(s). Dr. Hiroshi Nakanishi, Kyushu University, Japan 